\title{
Accuracy of phenotypic methicillin susceptibility methods in the detection of Staphylococcus aureus isolates carrying different SCCmec types
}

\author{
Pricilla DM de Matos, Ricardo P Schuenck, Fernanda S Cavalcante, \\ Roberta MF Caboclo, Kátia Regina N dos Santos/ ${ }^{+}$
}

Departamento de Microbiologia Médica, Instituto de Microbiologia, Universidade Federal do Rio de Janeiro, Rio de Janeiro, RJ, Brasil

A total of 138 isolates, 118 methicillin-resistant Staphylococcus aureus (MRSA) isolates (staphylococcal cassette chromosome type II, 20 isolates, type III, 39 isolates and type IV, 59 isolates) and 20 methicillin-sensitive S. aureus isolates were evaluated by phenotypic methods: cefoxitin and oxacillin disk diffusion (DD), agar dilution (AD), latex agglutination (LA), oxacillin agar screening (OAS) and chromogenic agar detection. All methods showed 100\% specificity, but only the DD tests presented 100\% sensitivity. The sensitivity of the other tests ranged from $82.2 \%$ (OAS)-98.3\% (AD). The LA test showed the second lowest sensitivity (86.4\%). The DD test showed high accuracy in the detection of MRSA isolates, but there was low precision in the detection of type IV isolates by the other tests, indicating that the genotypic characteristics of the isolates should be considered.

Key words: Staphylococcus aureus - SCCmec types - phenotypic methods

Methicillin-resistant Staphylococcus aureus (MRSA) has become a major epidemiological and clinical problem over the several last decades (Aires de Sousa \& de Lencastre 2004). Methicillin resistance is encoded by the $m e c A$ gene that is located in a mobile genetic element called the staphylococcal cassette chromosome (SCCmec) (Katayama et al. 2000). The analysis of SCCmec has revealed eight different allotypes, which are designated types I-VIII. Worldwide, most hospital-derived isolates belong to types I, II and III, most communityderived isolates belong to type IV (Chambers \& Deleo 2009). In Brazil, only isolates belonging to types II, III and IV have been found in hospitals (Reinert et al. 2008, Schuenck et al. 2009, Silva-Carvalho et al. 2009).

The reliable detection of methicillin resistance in clinical $S$. aureus isolates is essential for the appropriate treatment and epidemiological control of MRSA infections. In general, conventional phenotypic methods have shown high sensitivity and specificity in the detection of MRSA isolates (Velasco et al. 2005, Baddour et al. 2007). However, the emergence of $S$. aureus isolates carrying the SCCmec type IV allotype, which is associated with a minimum inhibitory concentration (MIC) for methicillin near the breakpoint (Schuenck et al. 2009), could influence the detection of this resistance. Here, we present the first study to evaluate the accuracy of different phenotypic methods in the detection of MRSA strains carrying different $\mathrm{SCCmec}$ types, using isolates from hospitals in Rio de Janeiro.

Financial support: FAPERJ, CNPq, CAPES, FUJB, PRONEX

+ Corresponding author: santoskrn@micro.ufrj.br

Received 26 April 2010

Accepted 12 August 2010
One hundred and thirty-eight isolates of $S$. aureus, including 118 MRSA and 20 methicillin-sensitive $S$. aureus (MSSA) isolates, were obtained from different clinical specimens (nostrils, $\mathrm{n}=33$; blood, $\mathrm{n}=31$; respiratory specimen, $n=30$; other sites, $n=44$ ) from patients in five hospitals in Rio de Janeiro between August 1999-July 2008. The isolates were identified according to Bannerman and Peacock (2007). The mecA gene and the SCCmec types were detected according to Ferreira et al. (2003) and to Oliveira and de Lencastre (2002), respectively.

The susceptibilities to cefoxitin $(30 \mu \mathrm{g})$ and oxacillin $(1 \mu \mathrm{g})$ (Oxoid, Basingstoke, England) were determined by the disk diffusion (DD) method on Mueller-Hinton agar (Difco Laboratories, Detroit, USA) according to the Clinical and Laboratory Standards Institute National Committee for Clinical Laboratory Standards (CLSI/ NCCLS) protocol (CLSI/NCCLS 2009a, c). S. aureus ATCC 25923 was used as the control strain.

The MIC of oxacillin (Sigma Chemical Company, St. Louis, MO, USA) was determined by the agar dilution (AD) method (CLSI/NCCLS 2009b, c). Briefly, bacterial suspensions were adjusted to a 0.5 McFarland standard, diluted $1: 10$ and inoculated ( $10^{4}$ colony-forming unit) on Mueller-Hinton agar (Difco) plates with $2 \%$ (wt $/ \mathrm{vol}$ ) $\mathrm{NaCl}$ and varying oxacillin concentrations $(0.5-256 \mu \mathrm{g} /$ $\mathrm{mL}$ ). The plates were incubated at $35^{\circ} \mathrm{C}$ for 24 h. S. aureus ATCC 29213 was used as the control strain.

All isolates were plated using a $0.5 \mathrm{McF}$ arland bacterial suspension on Mueller-Hinton agar (Difco) with $4 \%$ (wt/vol) $\mathrm{NaCl}$ and oxacillin (Sigma) at either 4 or $6 \mu \mathrm{g} / \mathrm{mL}$ (CLSI/NCCLS 2009b). Oxacillin resistance was confirmed by the presence of bacterial growth after $24 \mathrm{~h}$ and $48 \mathrm{~h}$ of incubation at $35^{\circ} \mathrm{C}$ (CLSI/NCCLS 2009c). $S$. aureus ATCC 29213 (susceptible) and S. aureus ATCC 33591 (resistant) were included as control strains.

Isolates were grown on chromogenic agar that is selective for MRSA, known as CHROMagar (Microbiol- 
ogy, Paris, France) (Diederen et al. 2005). The composition of this medium is proprietary. A 0.5 McFarland bacterial suspension was used to inoculate the chromogenic medium using a cotton swab and the tests were read at two time points: after $24 \mathrm{~h}$ and $48 \mathrm{~h}$ of incubation at $35^{\circ} \mathrm{C}$. The growth of pink/purple-clear colonies was indicative of the presence of MRSA isolates. S. aureus ATCC 29213 (oxacillin susceptible) and S. aureus ATCC 33591 (oxacillin resistant) were included as controls.

The latex agglutination (LA) test (Slidex MRSA Detection test; bioMérieux S/A, Paris, France) was performed according to the manufacturer's instructions. A loop full of colonies obtained from blood agar was suspended in $200 \mu \mathrm{L}$ of extraction reagent $\mathrm{n}^{\circ} 1(0.1 \mathrm{M} \mathrm{NaOH})$ and boiled for $3 \mathrm{~min}$. Next, $50 \mu \mathrm{L}$ of extraction reagent $\mathrm{n}^{\circ} 2(0.5 \mathrm{M}$ $\mathrm{KH}_{2} \mathrm{PO}_{4}$ ) was added to the suspension and the components were mixed well. This mixture was centrifuged $(1,500 \mathrm{~g} /$ $5 \mathrm{~min}$ ) and $50 \mu \mathrm{L}$ of the supernatant was placed in each of the two circles of the test slide. Anti-penicillin binding protein 2a (PBP2a) monoclonal antibody-sensitized latex $(50 \mu \mathrm{L})$ was added to one of the circles and $50 \mu \mathrm{L}$ of negative control latex was added to the other circle. The presence of agglutination was visually evaluated after
3 min. S. aureus ATCC 33591 (positive) and 25923 (negative) were included as controls in the test.

The sensitivities, specificities and positive and negative predictive values of the assays for MRSA strains with different SCCmec types and for MSSA isolates are presented in Table I. All the tests showed 100\% specificity and positive predictive values, but only the DD test had $100 \%$ sensitivity. MRSA isolates with SCCmec IV showed discordant results for all other tests that were evaluated. The AD test showed $98.3 \%$ sensitivity. Oxacillin MICs ranged from $0.5 \mu \mathrm{g} / \mathrm{mL}->256 \mu \mathrm{g} / \mathrm{mL}$ for the 118 MRSA isolates evaluated. For type III isolates, the MIC values were $\geq 128 \mu \mathrm{g} / \mathrm{mL}$, whereas for $80 \%$ of type II isolates, the MICs were between $64-128 \mu \mathrm{g} / \mathrm{mL}$. For type IV strains, $88.1 \%$ of the isolates showed MICs between $4-32 \mu \mathrm{g} / \mathrm{mL}$ and two isolates had MICs of 0.5 and $2 \mu \mathrm{g} / \mathrm{mL}$, values that are lower than the breakpoint values. The oxacillin agar screening (OAS) test with $6 \mu \mathrm{g} /$ $\mathrm{mL}$ of oxacillin showed the lowest sensitivity $(82.2 \%)$ among all of the tests evaluated; this test was not able to detect 21 MRSA isolates after $24 \mathrm{~h}$ of incubation. OAS with $4 \mu \mathrm{g} / \mathrm{mL}$ of oxacillin and CHROMagar ${ }^{\mathrm{TM}}$ showed sensitivities of $88.1 \%$ and $94 \%$, respectively. When the

\section{TABLE I}

Sensitivities, specificities and predictive values for antimicrobial susceptibility methods in comparison with the staphylococcal cassette chromosome (SCCmec) types in 118 methicillin-resistant Staphylococcus aureus

(MRSA) and 20 methicillin-sensitive S. aureus (MSSA) isolates evaluated in this study

\begin{tabular}{|c|c|c|c|c|c|c|c|c|c|c|c|c|}
\hline \multirow[b]{4}{*}{ Methods } & \multicolumn{6}{|c|}{ Number of isolates } & & & & & & \\
\hline & \multicolumn{6}{|c|}{$\operatorname{MRSA}(\mathrm{n}=118)$} & & & \multirow{3}{*}{$\begin{array}{c}\text { Sensitivity }^{a} \\
\%\end{array}$} & \multirow{3}{*}{$\begin{array}{c}\text { Specificity }^{b} \\
\%\end{array}$} & \multirow{3}{*}{$\begin{array}{l}\mathrm{PPV}^{c} \\
\%\end{array}$} & \multirow{3}{*}{$\begin{array}{c}\mathrm{NPV}^{d} \\
\%\end{array}$} \\
\hline & \multicolumn{2}{|c|}{$\begin{array}{c}\text { SCCmec II } \\
(\mathrm{n}=20)\end{array}$} & \multicolumn{2}{|c|}{$\begin{array}{c}\text { SCCmec III } \\
(\mathrm{n}=39)\end{array}$} & \multicolumn{2}{|c|}{$\begin{array}{c}\text { SCCmec IV } \\
(\mathrm{n}=59)\end{array}$} & \multicolumn{2}{|c|}{$\begin{array}{l}\text { MSSA } \\
(\mathrm{n}=20)\end{array}$} & & & & \\
\hline & $\mathrm{P}$ & $\mathrm{FN}$ & $\mathrm{P}$ & $\mathrm{FN}$ & $\mathrm{P}$ & $\mathrm{FN}$ & $\mathrm{N}$ & $\mathrm{FN}$ & & & & \\
\hline DD & 20 & - & 39 & - & 59 & - & 20 & - & 100 & 100 & 100 & 100 \\
\hline $\mathrm{AD}(\mathrm{MIC})$ & 20 & - & 39 & - & 57 & 2 & 20 & - & 98.3 & 100 & 100 & 90.9 \\
\hline LA & 20 & - & 39 & - & 43 & 16 & 20 & - & 86.4 & 100 & 100 & 55.5 \\
\hline \multicolumn{13}{|l|}{ OAS } \\
\hline \multicolumn{13}{|l|}{$4 \mu \mathrm{g} / \mathrm{mL}$} \\
\hline $24 \mathrm{~h}^{e}$ & 20 & - & 39 & - & 45 & 14 & 20 & - & 88.1 & 100 & 100 & 58.8 \\
\hline $48 \mathrm{~h}^{e}$ & 20 & - & 39 & - & 54 & 5 & 20 & - & 95.7 & 100 & 100 & 80 \\
\hline \multicolumn{13}{|l|}{$6 \mu \mathrm{g} / \mathrm{mL}$} \\
\hline $24 \mathrm{~h}$ & 20 & - & 39 & - & 38 & 21 & 20 & - & 82.2 & 100 & 100 & 48.8 \\
\hline $48 \mathrm{~h}$ & 20 & - & 39 & - & 52 & 7 & 20 & - & 94 & 100 & 100 & 74 \\
\hline \multicolumn{13}{|c|}{ CHROMagar } \\
\hline $24 \mathrm{~h}$ & 20 & - & 39 & - & 52 & 7 & 20 & - & 94 & 100 & 100 & 74 \\
\hline $48 \mathrm{~h}$ & 20 & - & 39 & - & 55 & 4 & 20 & - & 96.6 & 100 & 100 & 83.3 \\
\hline
\end{tabular}

$a$ : number of true positive results by the test/number of MRSA isolates; $b$ : number of true negative results by the test/number of MSSA isolates; $c$ : positive predictive value (PPV) (number of true positive results by the test/total number of positive results by testing); $d$ : negative predictive value (NPV) (number of true negative results by the test/total number of negative results by the test); $e$ : incubation period; AD: agar dilution; DD: disk diffusion; F: false-negative; LA: latex agglutination; MIC: minimum inhibitory concentration; N: negative; OAS: oxacillin agar screening; P: positive. 
TABLE II

Profiles presented by 31 methicillin-resistant Staphylococcus aureus isolates with staphylococcal cassette chromosome IV that were discordant by one or more oxacillin susceptibility phenotypic methods

\begin{tabular}{|c|c|c|c|c|c|c|c|c|}
\hline \multirow{3}{*}{$\begin{array}{l}\text { Isolates } \\
\mathrm{n}\end{array}$} & \multirow[b]{3}{*}{$\mathrm{AD}(\mathrm{MIC})$} & \multirow[b]{3}{*}{ LA } & \multirow{2}{*}{\multicolumn{2}{|c|}{$\begin{array}{c}\text { OAS } \\
(4 \mu \mathrm{g} / \mathrm{mL})^{a}\end{array}$}} & \multirow{2}{*}{\multicolumn{2}{|c|}{$\begin{array}{c}\text { OAS } \\
(6 \mu \mathrm{g} / \mathrm{mL})^{a} \\
\text { Incubation }\end{array}$}} & \multirow{2}{*}{\multicolumn{2}{|c|}{$\begin{array}{c}\text { CHROMagar } \\
\text { Incubation }\end{array}$}} \\
\hline & & & & & & & & \\
\hline & & & $24 \mathrm{~h}$ & $48 \mathrm{~h}$ & $24 \mathrm{~h}$ & $48 \mathrm{~h}$ & $24 \mathrm{~h}$ & $48 \mathrm{~h}$ \\
\hline 9 & $8-256(\mathrm{R})$ & - & + & + & + & + & + & + \\
\hline 5 & $4-32(\mathrm{R})$ & + & + & + & - & + & + & + \\
\hline 3 & 8-16 (R) & - & - & + & - & + & + & + \\
\hline 3 & $8(\mathrm{R})$ & + & - & + & - & + & + & + \\
\hline 2 & $8(\mathrm{R})$ & + & - & - & - & - & - & - \\
\hline 1 & $16(\mathrm{R})$ & + & - & + & - & + & - & + \\
\hline 1 & $16(\mathrm{R})$ & - & + & + & - & - & + & + \\
\hline 1 & $8(\mathrm{R})$ & - & - & - & - & - & - & - \\
\hline 1 & $8(\mathrm{R})$ & + & - & - & - & - & + & + \\
\hline 1 & $8(\mathrm{R})$ & - & - & + & - & - & + & + \\
\hline 1 & $8(\mathrm{R})$ & - & + & + & - & + & + & + \\
\hline 1 & $8(\mathrm{R})$ & + & + & + & + & + & - & + \\
\hline 1 & $2(\mathrm{~S})$ & + & - & + & - & + & - & + \\
\hline 1 & $0.5(\mathrm{~S})$ & + & - & - & - & - & - & - \\
\hline
\end{tabular}

a: Clinical and Laboratory Standards Institute interpretation categories; AD: agar dilution; LA: latex agglutination; MIC: minimum inhibitory concentration in $\mu \mathrm{g} / \mathrm{mL}$; OAS: oxacilin agar screening; R: resistant; S: susceptible; +: resistance result; -: sensibility result.

incubation period was extended to $48 \mathrm{~h}$, the sensitivity values for the $6 \mu \mathrm{g} / \mathrm{mL}$ OAS test, the $4 \mu \mathrm{g} / \mathrm{mL}$ OAS test and the CHROMagar ${ }^{\mathrm{TM}}$ test increased to $94 \%, 95.7 \%$ and $96.6 \%$, respectively. When using a $24-\mathrm{h}$ incubation, $35.6 \%$, $23.7 \%$ and $11.8 \%$ of type IV MRSA isolates showed no growth in the $6 \mu \mathrm{g} / \mathrm{mL}$ OAS test, the $4 \mu \mathrm{g} / \mathrm{mL}$ OAS test and the CHROMagar test, respectively. After $48 \mathrm{~h}$ of incubation, the percentages of non-growing type IV isolates were $11.9 \%, 8.5 \%$ and $6.8 \%$, respectively. Non-confluent growth was observed for $10.2 \%, 13.6 \%$ and $6.8 \%$ of isolates. Table II shows the results for the $31(52.5 \%)$ type IV MRSA isolates that were discordant with respect to at least one susceptibility test. Nine isolates gave false sensitivity results for the LA test, the second least accurate method for the detection of MRSA isolates used in the present study.

In this study, we evaluated the sensitivity of five phenotypic methods in the detection of MRSA isolates carrying SCCmec types II, III and IV allotypes and we verified that all the tests had $100 \%$ specificity. Moreover, the DD test using cefoxitin or oxacillin detected all MRSA isolates, showing high accuracy in MRSA detection. Although CLSI/NCCLS has just recommended the cefoxitin disk instead of the oxacillin disk for the detection of MRSA, in this study we verified that both disks were able to identify all 118 isolates, including those carrying the SCCmec type IV allotype. This is an interesting finding, as the DD test is the simplest and least expensive assay to detect MRSA isolates. Because of the simplicity and affordability of the DD test, all 118 MRSA isolates used in this study can be easily detected in any standard clinical microbiology laboratory.
Our MIC results correctly classified all isolates with SCCmec types II and III as methicillin resistant. Despite the fact that two type IV isolates were found to be sensitive to oxacillin suing the AD test, this test had a high overall sensitivity for the detection of MRSA (98.3\%), as has been reported by other authors (Swenson et al. 2005, Velasco et al. 2005). However, neither of these studies investigated the different SCCmec types.

The OAS with $6 \mu \mathrm{g} / \mathrm{mL}$ of oxacillin has been recommended by CLSI/NCCLS to confirm ambiguous results obtained by the DD test. It has been shown by other studies that there is a high concordance between the results of OAS and the results of genotypic methods for the detection of MRSA (Felten et al. 2002, Velasco et al. 2005). Nevertheless, in our study, OAS showed the lowest sensitivity (82.2\%), with one-third of type IV isolates going undetected. Moreover, a few type IV isolates presented non-confluent growth on this medium, that is, they showed heteroresistance to methicillin. This fact could explain the low sensitivity observed for the other tests used in this study. The lower concentration of oxacillin $(4 \mu \mathrm{g} / \mathrm{mL})$ was also used to determine if using a lower concentration would reduce the percentage of MRSA isolates that were undetected. Even at the lower oxacillin concentration, almost $1 / 4$ of type IV isolates were not detected, giving the test a sensitivity of $88.1 \%$.

CHROMagar $^{\mathrm{TM}}$ MRSA is a chromogenic medium used to detect MRSA isolates. The advantage of this medium is that it allows the presumptive identification of bacterial species and their methicillin resistance profiles in a single step. Recent studies have found that this test has a high sensitivity, up to $95.4 \%$ (Diederen et al. 2005) or $96 \%$ 
(Tandé et al. 2008). In our study, CHROMagar ${ }^{\mathrm{TM}}$ MRSA performed better (sensitivity of $94 \%$ ) than the OAS test, with a number of isolated remaining undetected being three times smaller.

The slidex MRSA detection test, which involves checking for the presence of the mecA gene product (PBP2a), has been used as a rapid method for the detection of methicillin resistance. In our study, this test presented the second lowest sensitivity (86.4\%) as a result of classifying 16 type IV isolates as sensitive. Recently, Akcam et al. (2009) used the latex technique to analyze 60 MRSA isolates and they found a similar sensitivity of $88.3 \%$. When we performed the latex test again with the bacterial growth from the edge of the cefoxitin inhibition halo, these results became positive, showing that the isolates were not expressing the gene products that result in resistance to methicillin. In another study from our group (Ferreira et al. 2003), we verified the influence of resistance induction in this test by analyzing coagulasenegative staphylococci isolates; for these experiments, we observed an increase in sensitivity from $97-99 \%$.

In the present study, we verified that the susceptibility results were influenced by the SCCmec IV allotypes. The presence of the type IV allotype, which causes a low level of methicillin resistance, resulted in the most discordant results. According to Memmi et al. (2008), an increased sensitivity to $\beta$-lactams in type IV isolates is associated with the loss of PBP4, which is responsible for mediating resistance to these drugs. To conclude, our results demonstrate the high accuracy of the cefoxitin and oxacillin DD tests in the detection of MRSA. Some isolates carrying the SCCmec IV allotype could not be detected by other phenotypic methods. Therefore, the interpretations of the results of MRSA studies should be carefully evaluated, taking into consideration the genotypic characteristics of the MRSA isolates analyzed.

\section{REFERENCES}

Aires de Sousa M, de Lencastre H 2004. Bridges from hospitals to the laboratory: genetic portraits of methicillin-resistant Staphylococcus aureus clones. FEMS Immunol Med Microbiol 40: 101-111.

Akcam FZ, Tinaz GB, Kaya O, Tigli A, Ture E, Hosoglu S 2009. Evaluation of methicillin resistance by cefoxitin disk diffusion and PBP2a latex agglutination test in mecA-positive Staphylococcus aureus, and comparison of $m e c A$ with femA, femB, femX positivities. Microbiol Res 164: 400-403.

Baddour MM, AbuElKheir MM, Fatani AJ 2007. Comparison of $m e c$ A polymerase chain reaction with phenotypic methods for the detection of methicillin-resistant Staphylococcus aureus. Curr Microbiol 55: 473-509.

Bannerman TL, Peacock SJ 2007. Staphylococcus, Micrococcus and other catalase-positive cocci. In PR Murray, EJ Barron, JH Jorgensen, MA Pfaller, ML Landry (eds.), Manual of clinical microbiology, 9th ed., ASM Press, Washington, p. 390-411.

Chambers HF, Deleo FR 2009. Waves of resistance: Staphylococcus aureus in the antibiotic era. Nat Rev Microbiol 7: 629-641.

CLSI/NCCLS - Clinical and Laboratory Standard Institute/National Committee for Clinical Laboratory Standards 2009a. Performance standards for antimicrobial disk susceptibility testing. Approved standards M02-A10, 10th ed., CLSI, Wayne, 56 pp.
CLSI/NCCLS - Clinical and Laboratory Standard Institute/National Committee for Clinical Laboratory Standards 2009b. Methods for dilution antimicrobial susceptibility tests for bacteria that grow aerobically. Approved standards M07-A8, 8th ed., CLSI, Wayne, 68 pp.

CLSI/NCCLS - Clinical and Laboratory Standard Institute/National Committee for Clinical Laboratory Standards 2009c. Performance standards for antimicrobial susceptibility testing. Approved standards M100-S19, 19th Informational Supplement, CLSI, Wayne, 152 pp.

Diederen B, van Duijn I, van Belkum A, Willemse P, van Keulen P, Kluytmans J 2005. Performance of CHROMagar MRSA medium for detection of methicillin-resistant Staphylococcus aureus. J Clin Microbiol 43: 1925-1927.

Felten A, Grandry B, Lagrange PH, Casin I 2002. Evaluation of three techniques for detection of low-level methicillin-resistant Staphylococcus aureus (MRSA): a disk diffusion method with cefoxitin and moxalactam, the Vitek 2 system, and the MRSA-screen latex agglutination test. J Clin Microbiol 40: 2766-2771.

Ferreira RB, Iorio NL, Malvar KL, Nunes AP, Fonseca LS, Bastos CC, Santos KR 2003. Coagulase-negative staphylococci: comparison of phenotypic and genotypic oxacillin susceptibility tests and evaluation of the agar screening test by using different concentrations of oxacillin. J Clin Microbiol 41: 3609-3614.

Katayama Y, Ito T, Hiramatsu K 2000. A new class of genetic element, staphylococcus cassette chromosome mec, encodes methicillin resistance in Staphylococcus aureus. Antimicrob Agents Chemother 44: 1549-1555.

Memmi G, Filipe SR, Pinho MG, Fu Z, Cheung A 2008. Staphylococcus aureus PBP4 is essential for beta-lactam resistance in community-acquired methicillin-resistant strains. Antimicrob Agents Chemother 52: 3955-3966.

Oliveira DC, de Lencastre H 2002. Multiplex PCR strategy for rapid identification of structural types and variants of mec element in methicillin-resistant Staphylococcus aureus. Antimicrob Agents Chemother 46: 2155-2161.

Reinert C, McCulloch JA, Watanabe S, Ito T, Hiramatsu K, Mamizuka EM 2008. Type IV SCCmec found in decade old Brazilian MRSA isolates. Braz J Infect Dis 12: 213-216.

Schuenck RP, Nouér SA, Winter C de O, Cavalcante FS, Scotti TD, Ferreira AL, Giambiagi-de Marval M, dos Santos KR 2009. Polyclonal presence of non-multiresistant methicillin-resistant Staphylococcus aureus isolates carrying SCCmec IV in health care-associated infections in a hospital in Rio de Janeiro, Brazil. Diagn Microbiol Infect Dis 64: 434-441.

Silva-Carvalho MC, Bonelli RR, Souza RR, Moreira S, dos Santos LC, de Souza Conceição M, de Mello Junior SJ, Carballido JM, Rito PN, Vieira VV, Teixeira LA, Sá Figueiredo AM 2009. Emergence of multiresistant variants of the community-acquired methicillin-resistant Staphylococcus aureus lineage ST1-SCCmecIV in two hospitals in Rio de Janeiro, Brazil. Diagn Microbiol Infect Dis 65: 300-305.

Swenson JM, Tenover FC, Cefoxitin Disk Study Group 2005. Results of disk diffusion testing with cefoxitin correlate with presence of mecA in Staphylococcus spp. J Clin Microbiol 43: 3818-3823.

Tandé D, Garo B, Ansart S, Lejeune B 2008. Efficiency of CHROMagar-MRSA in detecting meticillin-resistant Staphylococcus aureus in a routine setting. J Hosp Infect 70: 388-389.

Velasco D, del Mar Tomas M, Cartelle M, Beceiro A, Perez A, Molina F, Moure R, Villanueva R, Bou G 2005. Evaluation of different methods for detecting methicillin (oxacillin) resistance in Staphylococcus aureus. J Antimicrob Chemother 55: 379-382. 